\title{
Geomagnetic Variations with Periods from 5 to 30 Years at Hong Kong
}

\author{
Po-Fang CHEN \\ Department of Geography and Geology, University of Hong Kong, Hong Kong
}

(Received December 1, 1979; Revised July 30, 1980)

The study of the long-period variations of the earth's magnetic field requires a great number of observatories having long records of continuous operation. $\mathrm{Ob}$ servation at Hong Kong was commenced by the Royal Observatory in 1884 and continued without a break until 1939 (NeIGHBour et al., 1974). The data from several dozen observatories had been used in analyses of the secular variations by many authors, e.g. Yukutake (1965), Bhargava and YacoB (1969), Currie (1973), Rivin (1974), Alldredge (1976, 1977), Courillot and Mouël (1976), Srivastava and ABBAS (1977). However they did not use the data of Hong Kong, except Rivin's article (1974). Rivin used the horizontal component of Au-Tau (Hong Kong) only, but his result was different from this paper. The details will be discussed later.

The coordinate of Au-Tau (Hong Kong) Royal Observatory is $114.2^{\circ} \mathrm{E}$ (Geographic Longitude) and $22.2^{\circ} \mathrm{N}$ (Geographic Latitude). This study utilizes the annual means of midnight values of the horizontal component $(H)$, the vertical component $(Z)$, and the declination $(D)$. In order to remove the major trend, a suitable filtering technique is required. In this paper, the data were first preconditioned by subtracting values of a fifth degree polynomial curve, which is the best-fitting curve to the annual mean values. The residual data were then fitted by a sum of trigonometric sine terms, and also were analysed by the maximum entropy method (MEM) (ULRYCH, 1972; ChEn and Stegen, 1974; ANDERSEN, 1974).

Figures 1 and 2 show the regression curves - fifth degree polynomials for $H$ and $Z$ respectively. The plots are the annual means of midnight values. The following equations are obtained for the best fitting curve to the annual values by the method of least squares.

$$
\begin{aligned}
& H=36132+3.94 \cdot t+6.0822 \cdot t^{2}-0.299237 \cdot t^{3}+0.00547556 \cdot t^{4}-0.0000344689 \cdot t^{5} \\
& Z=23059-17.39 \cdot t-2.6240 \cdot t^{2}+0.150205 \cdot t^{3}-0.00298376 \cdot t^{4}+0.0000204336 \cdot t^{5}
\end{aligned}
$$

reckoning $t$ from 1884 (while $t=0$ ). They are given in gammas.

Figure 3 shows the residuals of $H$, which is obviously found to have a pattern with a variation of about 20 years period. Applying the maximum entropy method to the residuals, we can detect the major spectral peaks. In this study detection is 


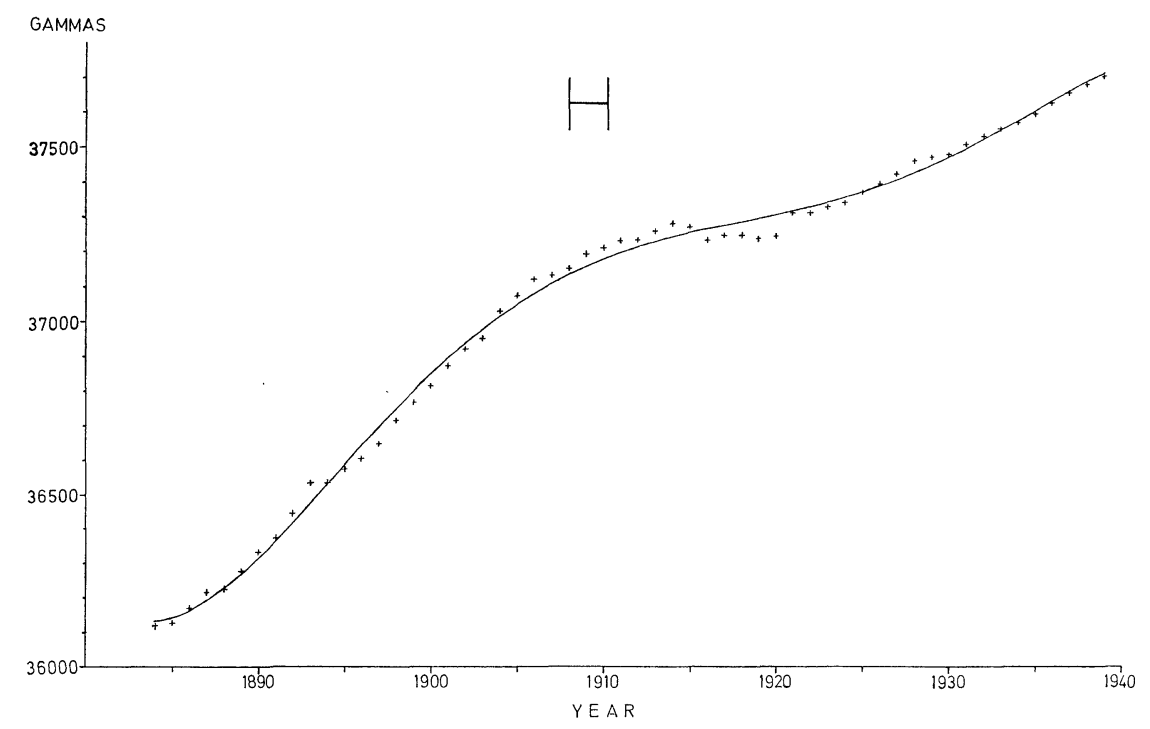

Fig. 1. Horizontal intensity at Hong Kong from 1884 to 1939. Crosses represent the actual annual midnight values. The smooth curve is the least-square-fit curve.

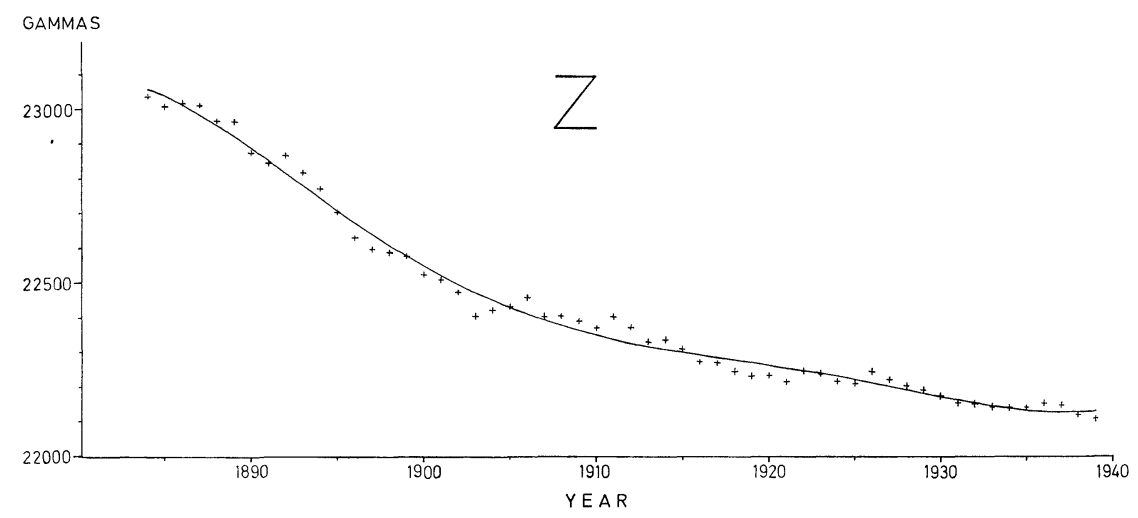

Fig. 2. Vertical intensity at Hong Kong from 1884 to 1939 . Crosses represent the actual annual midnight values. The smooth curve is the least-square-fit curve.

optimum when $50 \%$ of record length is used for the prediction error coefficient: that is a 28 point prediction error filter. Figure 4 shows the power spectrum of MEM. The significant spectral peaks are 20 years, 10.6 years, 7.1 years, and 5.8 years. These spectral peaks are consistent with the results of CURRIE (1973).

Similar to the above, Fig. 3 also shows the residuals of $Z$. A variation of about 20 years period can also be seen. When the maximum entropy method is used on the 


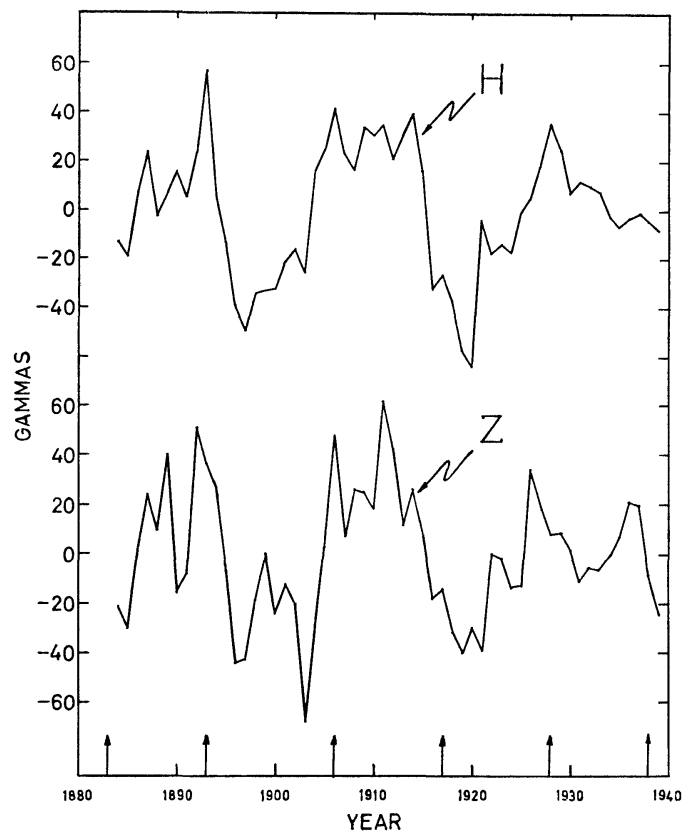

Fig. 3. Residuals of $H$ and $Z$ annual values from the least-square-fit curves. The vertical arrows show the sunspot maxima.

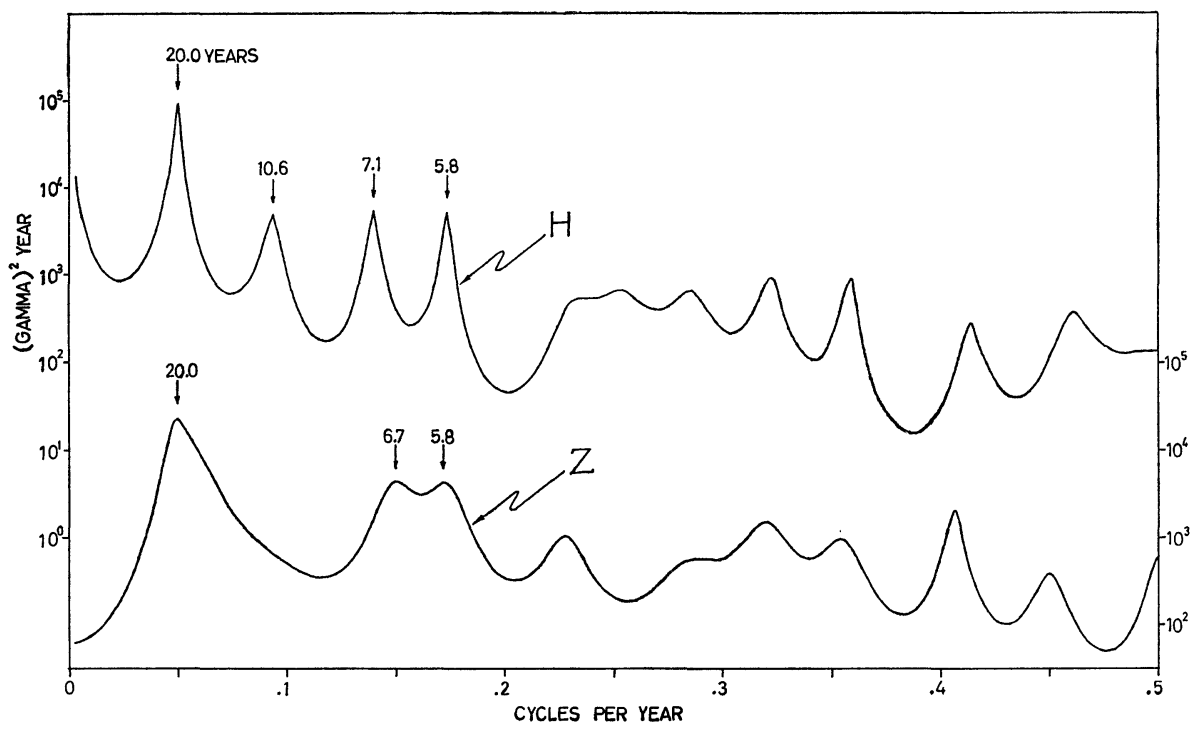

Fig. 4. Maximum entropy spectra of $H$ and $Z$ at Hong Kong. 


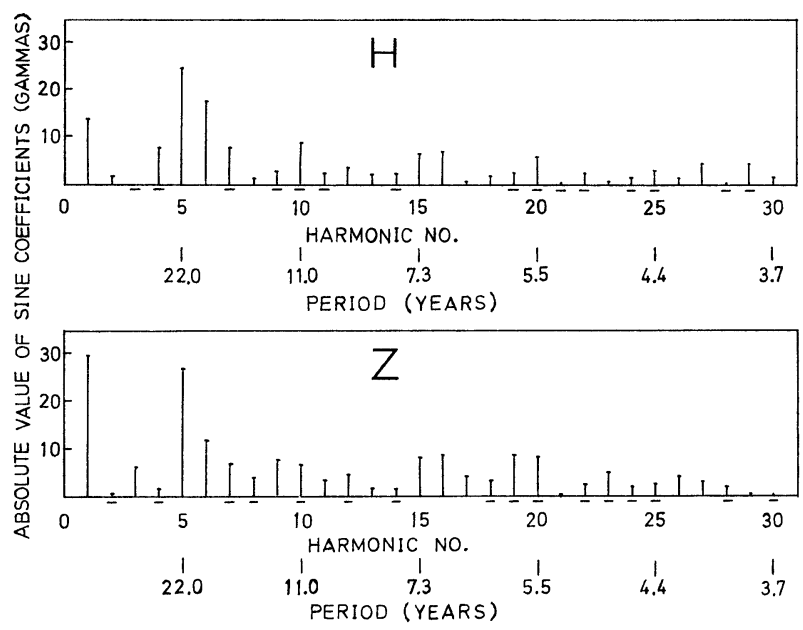

Fig. 5. Fourier coefficients for $H$ and $Z$ at Hong Kong. Minus sign under the vertical bar indicates a negative coefficient. All other coefficients are positive.

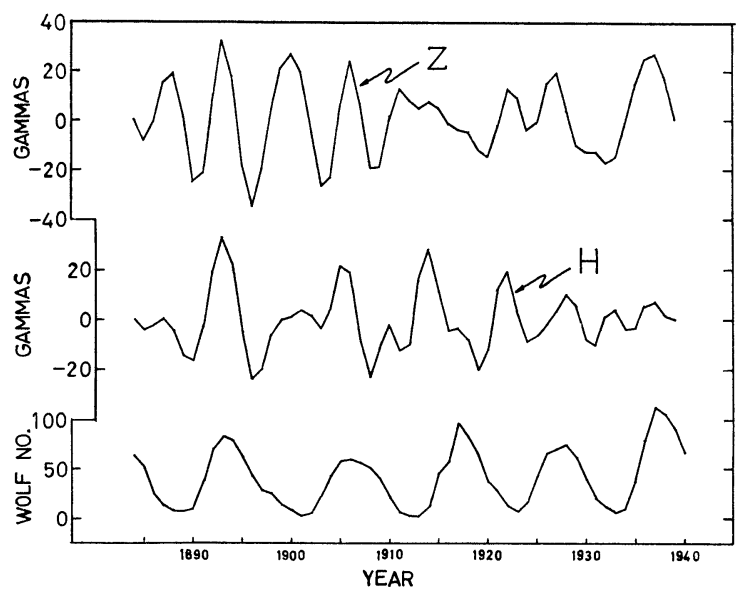

Fig. 6. Curves for $H$ and $Z$ synthesized from Fourier coefficients from harmonic numbers 9 to 30 . The relative sunspot numbers are shown at the bottom.

residuals data, the best prediction error filter is 26 points because the 28 point prediction error filter results in instability, i.e. the 20 years spectral peak is split in two peaks. Figure 4 also shows the power spectrum of $Z$. The significant spectral peaks are 20 years, 6.7 years, and 5.8 years. The absence of a solar cycle (10-11 years) is important for the analysis, which will be discussed in a later paragraph.

In order to compare the phase relation of these signals with the sunspot numbers, this paper used the method similar to AlldREDge (1976). The residual values were 
fit by a sum of trigonometric sine terms, the data being treated as an odd function with a fundamental period twice that of the observed interval. Then a function was synthesized from these Fourier coefficients by using all harmonics from 9 to 30 for both $H$ and $Z$. Figure 5 shows the Fourier coefficients for the residual data of $H$ and $Z$.

Let us discuss the results of $H$ first. Figure 6 shows the $H$ curve synthesized from Fourier coefficients from harmonic numbers 9 to 30 . The curve of sunspot numbers $W$ is also shown in Fig. 6. An out of phase relation can be seen, i.e. $H$ decreases as the sunspot numbers increase, but having two years lag. Roughly speaking, it was in agreement with the results of ALLDREDGE (1976). However, this result was different from Rivin's (1974). Rivin used the data of the same observatory (Au-Tau), but the method he used was double smoothing by a moving average, so it could be the reason that caused the difference. The amplitude of the solar cycle is 10.5 gammas, which was obtained by direct Fourier analysis following the application of a high-pass filter the same as YuKuTAKE's (1965) method. Then, if we put the amplitude of Hong Kong to the Fig. 4 of YUKUTAKE's article (1965), which concerned the distribution of the amplitude of the solar cycle of $H$ against the geomagnetic latitude, it would be favourable to the conclusion of YUKUTAKE (1965), i.e. $X$ component decreases as the latitude increases and has a maximum at the equator (see Fig. 8). In fact, the data of Hong Kong gives support to the hypothesis of an equatorial ring current as an external source. However, since there are only a few observatories located in the equatorial region, an explicit conclusion about the distribution of the amplitudes of the solar cycle variations of $H$ having a maximum at the equator still lacks reliable evidence. That is the reason why the data of Hong Kong, an observatory in the geomagnetic equatorial region, is valuable to the analysis.

Figure 6 also shows the $Z$ curve synthesized from Fourier coefficients from harmonic numbers 9 to 30 . It is evident that there is not any correlation between the variation of $Z$ and the sunspot numbers. This is consistent with the absence of the solar cycle spectral line in Fig. 4. In fact, it is an indispensable condition to the hypothesis of equatorial ring current, because the magnetic field comes from that current is parallel to the horizontal direction in the equatorial region, and the $Z$ component should be very small.

As to the period of 20 years, we can also synthesize Fourier coefficients from harmonic numbers 4 to 8 , and the curve is shown in Fig. 7. The most interesting phenomena is that the $H$ curve and the $Z$ curve are found to be exactly in phase (see Fig. 7), it implies that the variations of $H$ and $Z$ with 20 years period are caused by one origin. However, as compared with the results of ALLDREDGE (1977), we can really find that the curves of different observatories do not fit a common pattern recognizable as coming from a common sourse. Even if compared with the observatories in the equatorial region, e. g. Alibag, Honolulu, Apia, Mauritius, the patterns are different each and all. 


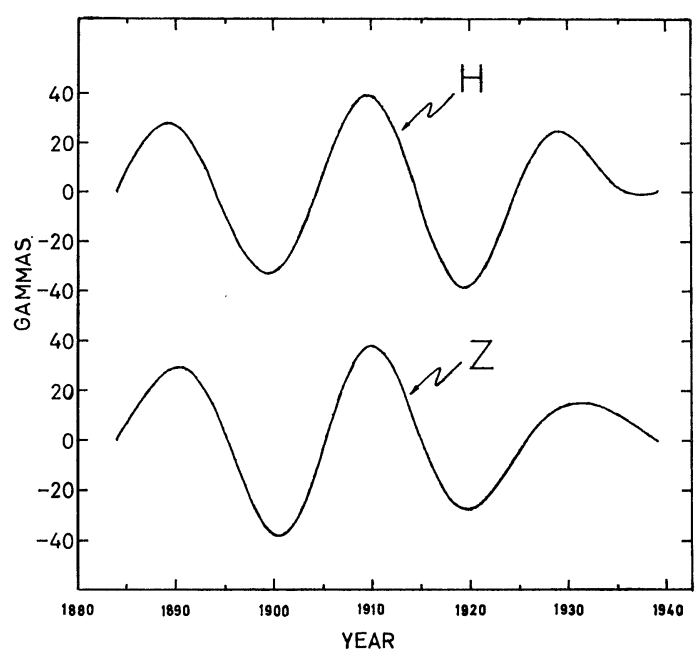

Fig. 7. Curves for $H$ and $Z$ synthesized from Fourier coefficients from harmonic numbers 4 to 8 .

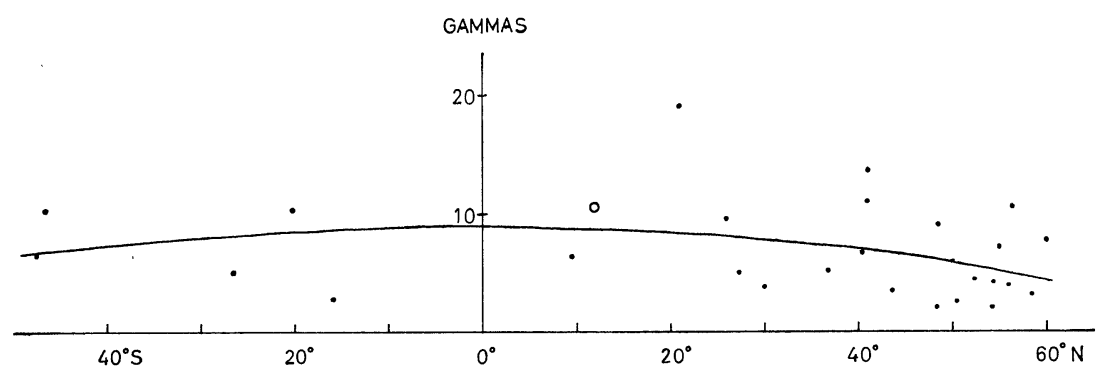

Fig. 8. Distribution of Fourier coefficients of the 11 years period against geomagnetic latitude. The circle represents the amplitude of Hong Kong. All other dots are based on the data in Table 4 of YUKUTAKE (1965).

Besides the two periods mentioned above, an oscillation period near 6.7 years exist both in $H$ component and $Z$ component (see Fig. 4) is noticeable. CURRIE (1973) pointed out this period near 6.7 years and related it to the free modes of the electromagnetically coupled core-mantle earth system, one of which has an oscillation period of about 6.7 years (YUKUTAKE, 1972). As a matter of course, one has to study in detail about the period of 6.7 years.

Lastly, deal with the result of $D$. The best fitting curve - fifth degree polynomial is

$$
D=35.9-2.490 \cdot t+0.09786 \cdot t^{2}-0.0050173 \cdot t^{3}+0.00009649 \cdot t^{4}-0.000000562 \cdot t^{5}
$$

reckoning $t$ from 1884 (while $t=0$ ). It is given in minutes. 


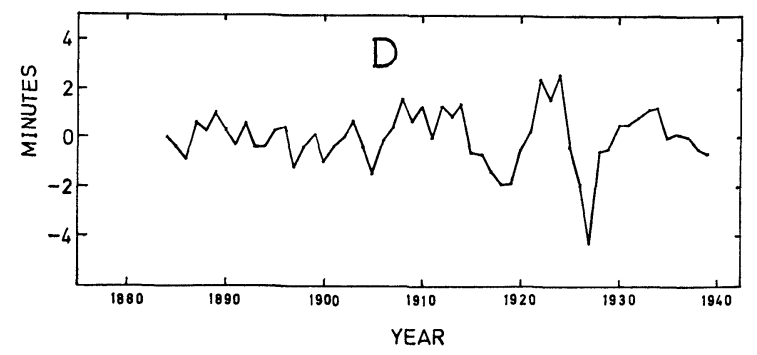

Fig. 9. Residuals of $D$ annual values from the least-square-fit curve.

The residuals of $D$ are shown in Fig. 9, which pattern seems to be more irregular. It had been analysed by using the maximum entropy method and the Fourier analysis method, but there was not any significant conclusion derived from the residuals.

\section{REFERENCES}

AlldREDGE, L. R., Effects of solar activity on geomagnetic component annual means, J. Geophys. Res., 81, 2990-2996, 1976.

ALLDREDGE, L. R., Geomagnetic variations with periods from 13 to 30 years, J. Geomag. Geoelectr., 29, 123-135, 1977.

ANDERSEN, N., On the calculation of filter coefficients for maximum entropy analysis, Geophysics, 39, 69-72, 1974.

BHARGAVA, B. N. and A. YACOB, Solar cycle response in the horizontal force of the earth's magnetic field, J. Geomag. Geoelectr., 21, 385-397, 1969.

Chen, W. Y. and G. R. STEGEN, Experiments with maximum entropy power spectra of sinusoids, J. Geophys. Res., 79, 3019-3022, 1974.

COURTILLOT, V. and J. L. Le MouËL, On the long-period variation of the earth's magnetic field from 2 months to 20 years, J. Geophys. Res., 81, 2941-2950, 1976.

CURRIE, R. G., Geomagnetic line spectra-2 to 7 years, Astrophys. Space Sci., 21, 425-438, 1973.

NeIGHbour, M. P., D. G. RIVERS, and G. O. WALKeR, The secular variation of components of the geomagnetic field as measured at Hong Kong from 1884-1973, J. Geomag. Geoelectr., 26, 443447, 1974.

Rivin, Yu. R., 11-year periodicity in the horizontal component of the geomagnetic field, Geomagn. Aeron., 14, 97-100, 1974 (Engl. Transl.).

SRIVASTAVA, B. J. and H. AbBas, Geomagnetic secular variation in India-regional and local features, J. Geomag. Geoelectr., 29, 51-64, 1977.

ULRYCH, T. J., Maximum entropy power spectrum of truncated sinusoids, J. Geophys. Res., 77, 1396$1400,1972$.

YUKUTAKE, T., The solar cycle contribution to the secular change in the geomagnetic field, J. Geomag. Geoelectr., 17, 287-309, 1965.

YUKUTAKE, T., The effect of change in the geomagnetic dipole moment on the rate of the earth's rotation, J. Geomag. Geoelectr., 24, 19-47, 1972. 